\title{
Erratum to: Immediate versus conventional loading of palatal implants in humans: a first report of a multicenter RCT
}

\author{
Britta A. Jung • Winfried Harzer • Heinrich Wehrbein • Tomasz Gedrange • \\ Werner Hopfenmüller • Gabriele Lüdicke • Maximilian Moergel • Peter Diedrich • \\ Martin Kunkel
}

Published online: 14 September 2010

(C) Springer-Verlag 2010

\section{Erratum to: Clin Oral Invest DOI 10.1007/s00784-010-0407-9}

At the time of data evaluation, one patient from the group that received immediate loading (BJ) was erroneously listed in the group that received conventional loading. However, the patient had been treated correctly according to randomization.

\begin{abstract}
Britta A. Jung and Martin Kunkel contributed equally to this work.
Trial registration Current Controlled Trials ISRCTN97142521.

Authors' contributions BJ and MK designed the study and served as the main contact persons for the sponsor and secondary study centers. BJ was in charge of the organization and will be responsible for the documentation of the study, which includes data collection, ethics committee approvals (all study centers), and orthodontic treatment (primary study center); BJ wrote the present manuscript. MK and MM are responsible for surgical implant insertion (primary study center). MK will be responsible for histomorphometric analyses. HW has a national and international reputation in skeletal anchorage. His entire experiences and consolidated knowledge concerning skeletal anchorage will be rendered accessible to the investigators (BJ, MK, HW, WH, PD, TG, GL) for the current research proposal. Werner Hopfenmüller (WH) performed the sample size calculation. All authors have read and approved the final manuscript.
\end{abstract}

The online version of the original article can be found at http://dx.doi. org/10.1007/s00784-010-0407-9.

B. A. Jung $\cdot$ H. Wehrbein

Department of Orthodontics, University Medical Center Mainz, Mainz, Germany

W. Harzer · G. Lüdicke

Department of Orthodontics, Technical University of Dresden, Dresden, Germany

T. Gedrange

Department of Orthodontics, University of Greifswald,

Greifswald, Germany

\section{W. Hopfenmüller}

Institute for Medical Statistics,

Charité-Campus Benjamin Franklin, Universtiy of Berlin,

Berlin, Germany
Therefore, at the interim evaluation the groups were composed of the following numbers after correction: according to the randomization protocol 21 implants were assigned to the group that received conventional loading (group 1) and 20 to the group that received immediate loading (group 2). The results of the study (such as implant loss and force magnitude) remained unaffected and are valid as published.

\footnotetext{
M. Moergel

Department of Oral and Maxillofacial Surgery,

University Medical Center Mainz,

Mainz, Germany

P. Diedrich

Department of Orthodontics, University of Aachen, Aachen, Germany

M. Kunkel

Department of Oral and Maxillofacial Plastic Surgery,

Ruhr-University of Bochum,

Bochum, Germany

B. A. Jung $(\bowtie)$

Department of Orthodontics, University Medical Center Mainz,

Augustusplatz 2, 55131 Mainz, Germany

e-mail: brjung@uni-mainz.de
} 